\title{
Colloid solution of surfactant monomers and polyelectrolyte: Polymerization and properties of the resulting interpolyelectrolyte complexes
}

\author{
N.V. Tsvetkov ${ }^{\text {a,* }}$, P.A. Fetin ${ }^{\text {b }}$, A.A. Lezov ${ }^{\text {a }}$, A.S. Gubarev ${ }^{\text {a }}$, L.I. Achmadeeva ${ }^{\text {a }}$, A.A. Lezova ${ }^{\text {a }}$, \\ I.M. Zorin ${ }^{\mathrm{b}}$, A.Yu. Bilibin ${ }^{\mathrm{b}}$ \\ a Department of Physics, St. Petersburg State University, Ulyanovskaya Street 1, St. Petersburg 198504, Russia \\ b Department of Chemistry, St. Petersburg State University, Universitetskij Prospect 26, St. Petersburg 198504, Russia
}

\section{A R T I C L E I N F O}

\section{Article history:}

Received 17 April 2015

Received in revised form 30 June 2015

Accepted 2 July 2015

Available online 20 July 2015

\section{Keywords:}

Surfactant monomer

Self-assembly

Polyelectrolyte

\begin{abstract}
A B S T R A C T
In the present work, we suggest a novel method for obtaining interpolyelectrolyte complex (IPEC) by free radical polymerization in water-salt dispersions of polyelectrolyte complexes (PECs) based on an anionic polyelectrolyte (sodium salt of poly (2-acrylamido-2-methyl-1-propanesulfonic acid) (PAMPS-Na)) and a cationic surfactant ((11-acryloyloxy) undecyl)-trimethylammonium bromide (AUTAB), as well as on PAUTAB cationic comblike polyelectrolyte and AMPS-Na monomer. IPEC consisting of PAMPS and PAUTAB was obtained by two ways. The first one is polymerization of surface active monomers AUTAB in the presence of PAMPS, and the second one is polymerization of AMPS monomers in the presence of PAUTAB. Molecular hydrodynamics methods were used to determine the molecular properties of initial components, polyelectrolyte complexes (PECS), and IPECs. Analysis of the dependence between translational friction coefficient of macromolecules and their molecular masses (which takes into account excluded-volume effects) allowed us to determine the Kuhn segment length of PAMPS-Na macromolecule $(A=6 \mathrm{~nm}$ ). Stable polyelectrolyte complexes, PEC, (PAMPS-AUTAB and PAUTAB-AMPS) were obtained by mixing solutions of components at low concentrations in equimolar ratios; polymerization of their low molecular weight components was carried out for the purpose of preparing IPECs. Comparison between scattered light intensity distributions measured for PEC and IPEC demonstrated that in IPEC solutions, only large aggregates of complex molecules are present, while in PEC solutions, two peaks were observed (those corresponding to diffusion of individual PECs and their aggregates). When concentrations of PEC components exceed $3.4 \mathrm{mM}$, polymerization results in the formation of IPEC precipitate which was studied by small angle X-ray scattering. It was found that the IPEC based on PAMPS-Na forms a film with hexagonal packing, and the PAUTAB-based IPEC forms a lamellar film.
\end{abstract}

(C) 2015 Elsevier B.V. All rights reserved.

\section{Introduction}

Interaction between polyelectrolytes and oppositely charged surfactants leads to the formation of polyelectrolyte complexes (PECs). The structure of PEC is influenced by forces of electrostatic interaction arising between oppositely charged fragments of polyelectrolyte and surfactant as well as by hydrophobic interactions between aliphatic surfactant tails [1]. Hydrophobic surfactant associates (the so-called intramolecular micelles) are formed on polyelectrolyte chain. In the condensed state, PECs may form liquid crystalline phases where these intramolecular micelles are arranged differently (lamellar, hexagonal or cubic packing) [2-6]. Binding between surfactant molecule and polyelectrolyte is accompanied by a cooperative effect which is observed

\footnotetext{
* Corresponding author.

E-mail address: n.tsvetkov@spbu.ru (N.V. Tsvetkov).
}

when surfactant concentration exceeds the critical aggregation concentration (CAC). This effect manifests itself in the dramatic increase in degree of surfactant-polyelectrolyte binding [7].

Typical values of CAC are several orders of magnitude lower than the critical micellar concentration (CMC) values for the corresponding surfactants [6]. After reaching stoichiometric composition of a complex, the $\zeta$-potential of PEC dispersions decreases, thus leading to the formation of a precipitate $[8,9]$; however, if the total concentration of PEC in the dispersion is lowered, precipitation can be avoided at stoichiometric ratio of the components [10].

Use of surface-active monomers provides the possibility of carrying out polymerization in nanostructured PEC ensembles at concentrations close to CAC. This polymerization yields an interpolyelectrolyte complex (IPEC). Radiation polymerization of PEC films in condensed state has been performed in $[11,12]$. The authors have demonstrated the possibility of fixating PEC structure by polymerization of surface-active monomers; however, high radiation doses led to destruction of the complex. 
<smiles>CCCCC(C)(C)NC(=O)C(C)(C)CS(=O)(=O)[O-]</smiles>

a

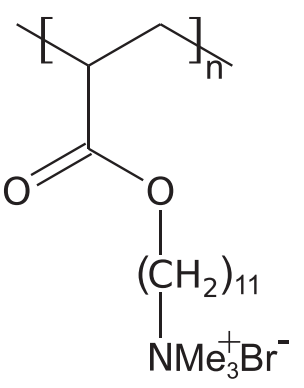

b
Fig. 1. Chemical structure of PAMPS-Na (a) and PAUTAB (b) macromolecules.

In the present work, we suggest a novel method for obtaining IPECs by free radical polymerization in water-salt dispersions of PECs based on an anionic polyelectrolyte (sodium salt of poly (2-acrylamido-2methyl-1-propanesulfonic acid), PAMPS-Na) and a cationic surfactant ((11-acryloyloxy) undecyl)-trimethylammonium bromide (AUTAB), as well as on PAUTAB cationic comb-like polyelectrolyte and AMPS-Na monomer. Several methods, such as dynamic light scattering, viscometry and velocity sedimentation, were used for determining molecular characteristics of PAMPS-Na and PAUTAB polymers and PEC (IPEC) complexes (in $0.05 \mathrm{M} \mathrm{NaCl}$ solutions). The samples of IPEC including PAMPS-Na were studied by atomic force microscopy (AFM) on mica substrate. Small-angle X-ray scattering was used for comparison between molecular packing of IPECs based on PAMPS-Na and PAUTAB.

\section{Experimental section}

\subsection{Synthesis of monomers}

The synthesis of ((11-acryloyloxy)undecyl)-trimethylammonium bromide (AUTAB) is described in detail elsewhere [13]. The authors used the two-stage method involving acylation of 11-bromoundecanol by acryloyl chloride, chromatographic isolation of the product and subsequent quaternization reaction (with $\mathrm{N}(\mathrm{Me})_{3}$ ). We used a similar technique in the synthesis of AUTAB; however, the use of freshly distilled $\mathrm{N}$-methylpyrrolidone as a solvent in acylation allowed us to replace the laborious stage of product isolation (preparative chromatography) with rather easy extraction using petroleum ether. Acylation was performed in the following manner: acryloyl chloride (15\% excess) distilled over $\mathrm{CuCl}$ at $73-74{ }^{\circ} \mathrm{C}$ was added to the solution of $3 \mathrm{~g}$ of 11 bromoundecanol (Aldrich, 98\%) in $25 \mathrm{~mL}$ of freshly distilled $\mathrm{N}$ methylpyrrolidone. The reaction was carried out at $-18{ }^{\circ} \mathrm{C}$ for $72 \mathrm{~h}$; then the reaction mixture was poured into $\mathrm{HCl}$ solution $(0.5 \mathrm{M})$. The product was extracted with petroleum ether and isolated after removing the solvent. In quaternization reaction, a polar solvent (acetonitrile) was used, this allowing shortening the reaction time from $53 \mathrm{~h}$ to $24 \mathrm{~h}$.

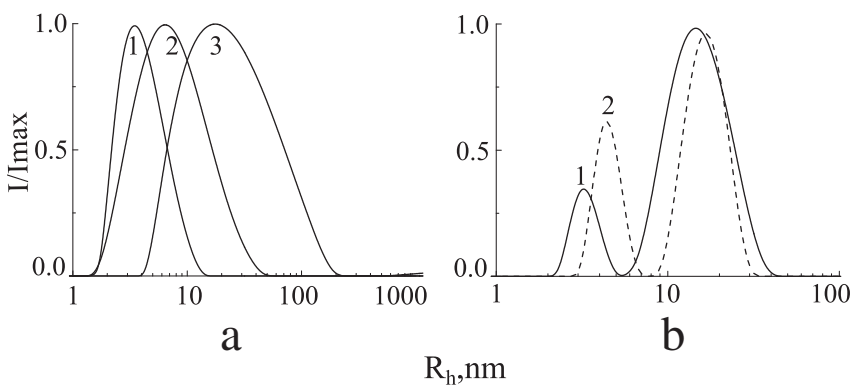

Fig. 2. Distributions of hydrodynamic radii obtained from the light scattering data for investigated samples dissolved in $0.05 \mathrm{M} \mathrm{NaCl}$ solution at a scattering angle of $90^{\circ}$. (a) corresponds to PAMPS-Na; (b) corresponds to PAUTAB-1 (solid line), PAUTAB-2 (dashed line). Curve numbers in the plots correspond to sample numbers in Table 1.

AMPS-Na monomer was obtained by $\mathrm{pH}$ titration of $0.1 \mathrm{M}$ water solution of AMPS-Na (Merck).

PAMPS-Na polyelectrolytes (see Fig. 1a) were prepared by free radical polymerization of AMPS-Na in aqueous solution. In all experiments, the "extra-pure grade" water with a resistivity of $16-18 \mathrm{M} \Omega \cdot \mathrm{m}$ was used (it was obtained using a UWOI-MF-1NA(18)-4 water purifier, Scientific and Production Corporation "Mediana Filter"). Low molecular weight impurities were removed from the prepared polymer by dialysis; the polymer was isolated by precipitation with acetone. In the case of PAUTAB polyelectrolytes (see Fig. 1b), the polymers were isolated by precipitation with dioxane and washed with diethyl ether. The solutions of PAMPS-Na and PAUTAB in $0.05 \mathrm{M} \mathrm{NaCl}$ were prepared at room temperature.

The ionic strength of solution can affect complex formation dramatically. The binding affinity between surfactants and opposite charged polyelectrolytes is reduced with the increase of the ionic strength but it increases the cooperative of the interaction [1]. Aqueous $0.05 \mathrm{M} \mathrm{NaCl}$ was used as "salt buffer" for complex formation in this work, such concentration is sufficient to eliminate large change in ionic strength of solution during the addition of the initiator. Also such concentration is sufficient to overcome the polyelectrolyte effects for PAMPS-Na macromolecules [34].

Polyelectrolyte complexes (PECs) of PAMPS-AUTAB and PAUTABAMPS composition were prepared by simple mixing equimolar amounts of monomer and polyelectrolyte in $0.05 \mathrm{M}$ aqueous solution of $\mathrm{NaCl}$. PEC polymerization was carried out in no less than $24 \mathrm{~h}$ after complex preparation in argon atmosphere $\left(C_{i}=1 \mathrm{~g} / \mathrm{L}, \mathrm{T}=60^{\circ} \tilde{\mathrm{N}}, 3 \mathrm{~h}\right)$ with $\mathrm{K}_{2} \mathrm{~S}_{2} \mathrm{O}_{8}$ and 2,2'-azobis(2-methylpropionamidine) dihydrochloride initiators (Aldrich) in the cases of PAMPS-AUTAB and PAUTAB-AMPS, respectively. If a precipitate was formed, the product was centrifuged, decanted and dried under vacuum using an oil pump; otherwise the complex was studied without isolation.

\subsection{Methods}

Size-exclusion chromatography (SEC) was performed using a Shimadzu LC-20 Prominence instrument (Shimadzu, Japan) equipped

Table 1

Molecular characteristics of PAMPS-Na and PAUTAB samples obtained using their solutions in $0.05 \mathrm{M} \mathrm{NaCl}$.

\begin{tabular}{|c|c|c|c|c|c|c|c|c|c|c|c|}
\hline Sample & $\begin{array}{l}{[\eta],} \\
\mathrm{cm}^{3} / \mathrm{g}\end{array}$ & $\mathrm{k}^{\prime}$ & $\begin{array}{l}D_{0} \times 10^{7}, \\
\mathrm{~cm}^{2} / \mathrm{s}\end{array}$ & $\begin{array}{l}\mathrm{S}, \\
\text { Sv }\end{array}$ & $M_{W} \times 10^{-3}$ & $\begin{array}{l}A_{2} \times 10^{4} \\
\mathrm{~mol} \mathrm{~g}^{2} \mathrm{~cm}^{3}\end{array}$ & $M_{S D} \times 10^{-3}$ & $M_{D \eta} \times 10^{-3}$ & $M_{W} / M_{N}$ & $\begin{array}{l}R_{h}, \\
\mathrm{~nm}\end{array}$ & $\mathrm{Z}^{\mathrm{a}}$ \\
\hline PAMPS-1 & 68 & 0.18 & 2.52 & 4.49 & 100 & 26.0 & 116 & 110 & 3.6 & 10 & 503 \\
\hline PAMPS-2 & 270 & 0.17 & 0.92 & 7.94 & 500 & 15.0 & 517 & 600 & 4.4 & 27 & 2240 \\
\hline PAMPS-3 & 450 & 0.28 & 0.67 & 10.29 & 900 & 9.5 & 1005 & 900 & 7.0 & 37 & 4352 \\
\hline PAUTAB-1 & 134 & 0.08 & $\begin{array}{l}2.22 \\
0.504^{\mathrm{b}}\end{array}$ & & & & 80 & & & 11 & 229 \\
\hline PAUTAB-2 & 122 & 0.08 & $\begin{array}{l}2.15 \\
0.669^{b}\end{array}$ & & & & 100 & & & 11 & 277 \\
\hline
\end{tabular}

\footnotetext{
a The degree of polymerization $Z$ was determined as a $M_{S D} / M_{0}$ ratio for PAMPS-Na macromolecules $\left(M_{0}\right.$ PAMPS $\left.=231\right)$ and as a $M_{D \eta} / M_{0}$ ratio for PAUTAB macromolecules $\left(M_{0}\right.$ PAUTAB $=$ 364).

b The scattered light distributions of PAUTAB solutions consisted of two peaks, with the second component having the corresponding diffusion coefficient.
} 


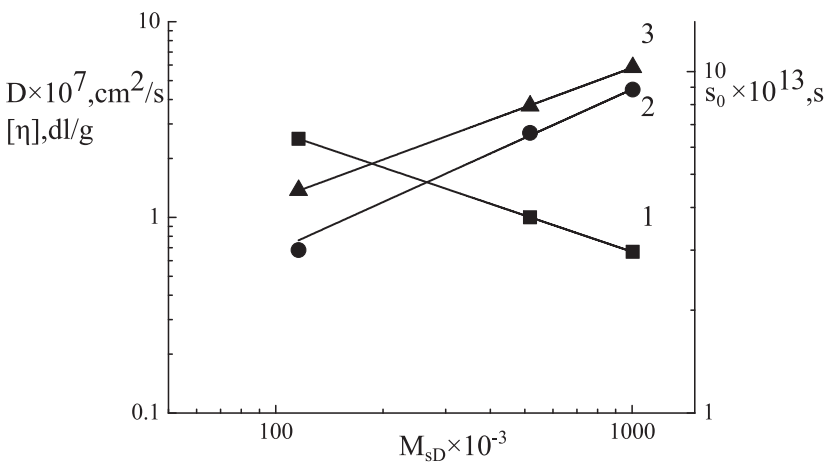

Fig. 3. Dependences of translational diffusion coefficient $D_{0}$ (1), intrinsic viscosity $[\eta](2)$ and sedimentation coefficient $s_{0}(3)$ on molecular mass $M_{S D}$ plotted on a double logarithmic scale (for PAMPS-Na samples in $0.05 \mathrm{M} \mathrm{NaCl}$ ).

with a TSKgel SuperAW5000 column and TSKgel SuperAW-H guard column (Supelco). $0.5 \mathrm{M} \mathrm{NaNO}_{3}$ solution was used as an eluent $\left(30^{\circ} \mathrm{C}\right.$ ); the elution rate was $0.5 \mathrm{~mL} / \mathrm{min}$. Calibration with the use of pullulan standards was made for these conditions. The experiments were performed using LCsolution software; the obtained results were subsequently processed by the GPC-Postrun Analysis program.

Dynamic light scattering (DLS) and static light scattering (SLS) are very efficient in the analysis of molecular characteristics of polymers, PECs and IPECs [8,14-20]. The "PhotoCor-Complex" setup (Photocor Instruments Inc., Moscow, Russia) was used in light scattering experiments; the spectrometer was equipped with a real-time correlator ( 288 channels, $10 \mathrm{~ns}$ ). Two lasers ( $\lambda_{0}=405$ and $654 \mathrm{~nm}$ ) were used as excitation sources; the experiments were carried out at scattering angles ranging from $30^{\circ}$ to $130^{\circ}$ at a temperature of $25 \pm 0.1^{\circ} \mathrm{C}$. Autocorrelation functions of scattered light intensity were processed by DynaLS software (providing the distributions of scattered light intensity and, therefore, distributions of relaxation times $\tau$ ). The dependence between $1 / \tau$ and the square of the scattering vector $q^{2}=\frac{4 \pi n_{0}}{\lambda_{0}} \sin (\theta / 2)$ for all studied samples was a line passing through the origin, indicating the diffusional character of the observed processes [21]. Translational diffusion coefficient $D$ was calculated from the slope of this line according to the following relationship:

$\frac{1}{\tau}=D q^{2}$

Hydrodynamic radius $R_{h}$ was calculated using the Stokes-Einstein equation [22]:

$R_{h}=\frac{k T}{6 \pi \eta_{0} D}$,

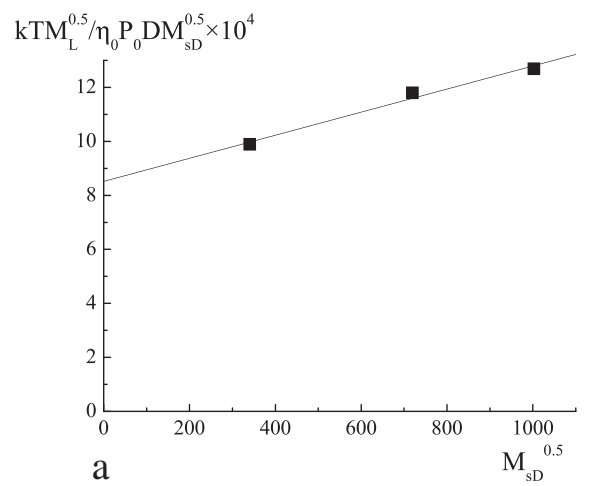

where $k=1.38 \times 10^{-23} \mathrm{~J} / \mathrm{K}$ is the Boltzmann constant, $T$ is the absolute temperature and $\eta_{0}$ is the solvent viscosity.

Weight-average molecular masses $M_{W}$ of PAMPS-Na samples dissolved in $0.05 \mathrm{M} \mathrm{NaCl}$ solution were determined from the data of static light scattering according to the following equation

$\left(\frac{H c}{R_{\theta}}\right)_{\theta \rightarrow 0}=\frac{1}{M_{W}}+2 A_{2} c$

where, $H=\frac{4 \pi^{2} n_{0}^{2}}{N_{A} \lambda_{0}^{4}}\left(\frac{\Delta n}{\Delta c}\right)^{2}, R_{\theta}$ is the Rayleigh relationship, and $A_{2}$ is the second virial coefficient [24].

Refraction indices of solutions $n$ and the solvent $n_{0}$ were determined using the RM40 automatic refractometer (Mettler Toledo, Switzerland). The value of refractive index increment of PAMPS-Na $\frac{\Delta n}{\Delta c}$ at $25{ }^{\circ} \mathrm{C}$ was $0.15 \mathrm{~cm}^{3} / \mathrm{g}$. $\zeta$-Potentials were determined using the SZ100 laser particle size analyzer (Horiba, Japan) equipped with a micro-electrophoretic cell.

Velocity sedimentation experiments were carried out on a Beckman XLI analytical ultracentrifuge (ProteomeLab XLI Protein Characterization System) equipped with the 4-hole titanium rotor at the speed of $40,000 \mathrm{rpm}$ and thermostated at $25^{\circ} \mathrm{C}$. An interference optical system (660 nm laser) was used and the rotor was loaded with double-sector cells holding epon-charcoal centerpieces of an optical path of $12 \mathrm{~mm}$. The concentration dependence of sedimentation coefficients was studied within a 3-fold concentration range of solution in order to determine the sedimentation coefficient $s_{0}$ at infinite dilution. Sedfit program allowed performing the analysis of the sedimentation velocity data, where a 'continuous $c(s)$ distribution model' was chosen for data acquisition $[25,26]$, which allows obtaining the sample distribution with respect to sedimentation coefficients using Provencher's regularization procedure $[27,28]$. The $s$ values in Svedberg units $\left(1 \times 10^{-13} s\right)$ at Table 1 are the weight average values of corresponding $c(s)$ distributions measured at three different concentrations and extrapolated to infinite dilution.

Viscosity of PAMPS-Na and PAUTAB samples was measured with the use of a Lovis $2000 \mathrm{M}$ rolling-ball microviscometer (Anton Paar, Austria) at $25^{\circ} \mathrm{C}$; the capillary inclination angle was varied from $55^{\circ}$ to $35^{\circ}$ at $5^{\circ}$ intervals. The obtained values of dynamic viscosity $\eta$ were averaged for a series of angles and used in calculations of reduced viscosity $\eta_{r}=\frac{\eta_{s}-\eta_{0}}{c \eta_{0}}$, where $c$ is the concentration of a solution. After extrapolation of reduced viscosity to infinite dilution, intrinsic viscosity $[\eta]$ of the polymer under study was determined using the relationship $\eta_{r}=[\eta]+k^{\prime}[\eta]^{2}+\ldots[23$, 24 ], where $k^{\prime}$ is the dimensionless Huggins constant. The constant $k^{\prime}$, which characterizes a polymer-solvent system, is dependent on the solvent quality and independent of the molar mass of dissolved polymer. In theta solvents it is close to $0.4-0.7$, in good solvents $k^{\prime} \simeq 0.2-0.4$ [29]. Density measurements were performed using the DM40 densitometer (Mettler Toledo, Switzerland).

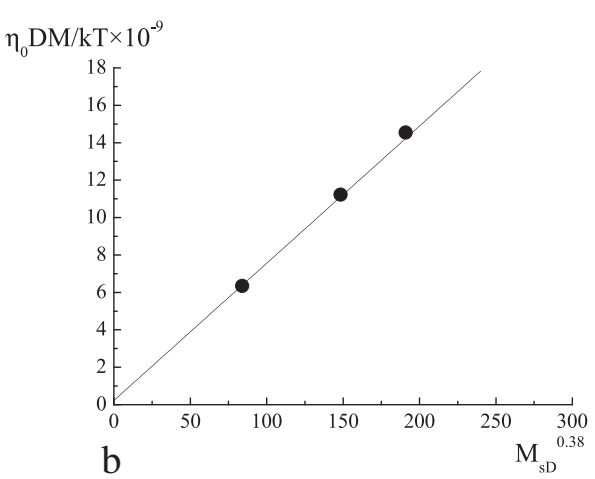

Fig. 4. Dependence between $k T M_{L}^{0.5} / \eta_{0} P_{\infty} M_{s D}^{0.5}$ and $M_{s D}^{0.5}$ (a); dependence between $\eta_{0} D M_{s D} / k T$ and $M_{s D}^{0.38}$ for PAMPS samples dissolved in $0.05 \mathrm{M} N a C l$ solution (b). 
AFM images were taken using a VEECO diNanoScope V instrument (Veeco Instruments, US) in semi-contact mode. Freshly cleaved mica sheets were used as a substrate. The mica substrate $(5 \times 5 \mathrm{~mm})$ was glued onto a metal plate and placed horizontally on the crosscut end of a motor shaft; $20 \mu \mathrm{L}$ of the studied solution was then placed onto this surface. The drop was exposed for $1 \mathrm{~min}$, then the motor was switched on for $1 \mathrm{~min}$ (rotation speed was $3000 \mathrm{rpm}$ ). The samples were dried in air for not less than $24 \mathrm{~h}$. The obtained images were processed with the aid of the NanoScope Analysis program.

Potentiometric measurements were conducted using the IPL-301 pH-meter/millivoltmeter (Multitest, Russia) equipped with the combined electrode for measuring $\mathrm{pH}$. Silver-chloride and thalliumchloride reference electrodes were used for ionometry. The surfactantselective electrode was made according to the technique described elsewhere [30].

\section{Results and discussion}

\subsection{Molecular characteristics of initial components of PECS and IPECS}

According to the SEC data, the synthesized anionic polyelectrolyte samples (PAMPS-Na) have rather high polydispersity indices $M_{W} / M_{N}$ (Table 1). The distributions of hydrodynamic radii obtained from the light scattering data for all PAMPS-Na samples (Fig. 2a) as well as sedimentation coefficient distributions demonstrated only one maximum.

Two samples of comb-like PAUTAB polymer were synthesized. Their hydrodynamic radius distributions include two peaks (Fig. 2b). The lower peak corresponds to the diffusion of individual molecules, and the appearance of the second peak may be attributed to the presence of intermolecular PAUTAB aggregates in the solution.

Concentration dependences of reduced viscosity and translational diffusion coefficient for PAMPS-Na and PAUTAB samples dissolved in $0.05 \mathrm{M} \mathrm{NaCl}$ solution were straight lines. Extrapolation of these lines to infinite dilution allowed determining the values of intrinsic viscosity $[\eta]$ and translational diffusion coefficient $D_{0}$ of the studied macromolecules (Table 1). Sedimentation coefficients of PAMPS-Na macromolecules in $0.05 \mathrm{M} \mathrm{NaCl}$ solution $\left(s_{0}\right)$ were obtained by extrapolation of linear concentration dependences of reciprocal sedimentation coefficient $s^{-1}$ to zero concentration.

Molecular masses of PAMPS-Na samples $\left(M_{S D}\right)$ were calculated from the values of sedimentation constants $s_{0}$ and translational diffusion coefficients $D_{0}$ by the following equation:

$M_{s D}=\frac{D_{0}}{s_{0}} \frac{k T N_{A}}{\left(1-\bar{v} \rho_{0}\right)}$.

The buoyancy factor $\left(1-\bar{v} \rho_{0}\right)=0.38$ was determined by comparing densities of solvent and solution at varied concentrations. Molecular masses were also determined by static light scattering. The results obtained by different methods were comparable (Table 1 ).

The presence of polymer aggregates in PAUTAB solutions rendered it difficult to use static light scattering for determination of PAUTAB molecular masses. Therefore, molecular masses of PAUTAB-1 and PAUTAB-2 samples were estimated using Eq. (5):

$M_{D \eta}=\left(\frac{A_{0} T}{\eta_{0} D}\right)^{3} \frac{1}{[\eta]}$

The chosen value of hydrodynamic invariant $A_{0}$ was $3.2 \times 10^{-17} \mathrm{~J} / \mathrm{K}$. This value of $A_{0}$ was given in [31] for macromolecules of close chemical structure; it is typical for flexible and moderately rigid polymers in good and ideal solvents [22]. Molecular masses of PAUTAB-1 and PAUTAB-2 samples turned out to be rather close (Table 1 ). In the synthesis of PAUTAB-based complexes PEC-2 and IPEC-2, sample 1 was used.

The dependences of intrinsic viscosity $[\eta]$, translational diffusion coefficient $D_{0}$, and sedimentation coefficient $s_{0}$ (see Fig. 3 ) on molecular

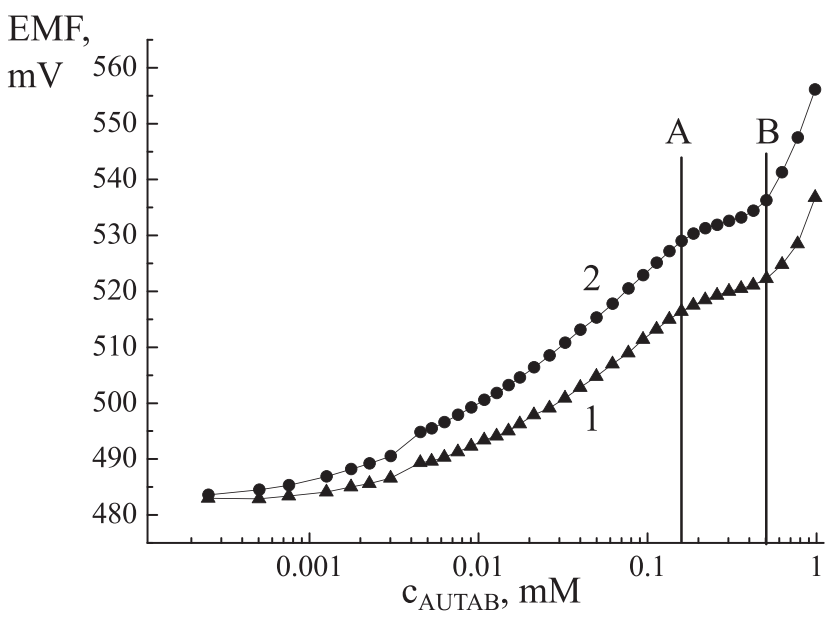

Fig. 5. Dependence of EMF in electrochemical cell containing aqueous solutions of PAMPSNa-1 (1) and PAMPS-Na-3 (2) ( $\mathrm{C}=0.77 \mathrm{mM})$ on AUTAB concentration. Polymer concentration is expressed in molar amount of monomer units per $1 \mathrm{~L}$ of the solution. The A point corresponds to the beginning of cooperative binding between AUTAB and PAMPS-Na; the $B$ point corresponds to saturation of polyelectrolyte with surfactant.

mass $M_{S D}$ for PAMPS-Na samples can be described by the corresponding Mark-Kuhn-Houwink equations (Eq. (6)).

$D_{0}=3.4 \times 10^{-4} \mathrm{M}^{-0.62} \mathrm{~cm}^{2} / \mathrm{s}$
$s_{0}=5.0 \times 10^{-15} \mathrm{M}^{0.38} \mathrm{~Sv}$
$[\eta]=5.1 \times 10^{-3} \mathrm{M}^{0.82} \mathrm{~cm}^{3} / \mathrm{g}$

High values of indices in the equations for intrinsic viscosity and diffusion coefficient indicate that $0.05 \mathrm{M} \mathrm{NaCl}$ solution is a thermodynamically good solvent for PAMPS-Na macromolecules. This fact is also evidenced by high values of second virial coefficients determined by static light scattering and values of $k^{\prime}$ [29] (Table 1).

To determine the Kuhn segment length $A$ of PAMPS-Na macromolecules in $0.05 \mathrm{M} \mathrm{NaCl}$ solution, the method proposed in [32] was used. The data points were extrapolated to the region of low molecular masses (where swelling caused by volume interactions tends to zero). The intrinsic translational friction coefficient may be expressed as $[f]=f / \eta_{0}$, where $\eta_{0}$ is the solvent viscosity $(\mathrm{P})$. Then, according to the Einstein equation, this formula can be rewritten as $[f]=\frac{k T}{\eta_{0} D}$. Using this expression as well as the value of molecular mass per unit length for PAMPS $\left(M_{L}=\frac{M_{0}}{\lambda}=9.2\right.$ $4 \times 10^{9} 1 / \mathrm{cm}$, where $M_{0}$ is the molecular mass, and $\lambda$ is the length of a repeating unit), we can obtain the following equation:

$\frac{k T}{\eta_{0} D} \frac{1}{P_{\infty}} \frac{M_{L}^{1 / 2}}{M^{1 / 2}}=A^{1 / 2}+0.201 B A^{-2} M_{L}^{1 / 2} M^{1 / 2}$

The $P_{\infty}$ constant is equal to 5.11 , and B is the parameter characterizing the polymer/solvent interaction.

The direct method to take volume interactions into account in the analysis of experimental data was proposed in [33] for translational

Table 2

Molecular characteristics of PEC- 1 and IPEC- 1 in $0.05 \mathrm{M} \mathrm{NaCl}$ at a concentration of 0.77 $\mathrm{mM}$.

\begin{tabular}{llllllll}
\hline Sample & Matrix & PEC/IPEC & $\begin{array}{l}D_{1} \times 10^{7}, \\
\mathrm{~cm}^{2} / \mathrm{s}\end{array}$ & $\begin{array}{l}D_{2} \times 10^{7}, \\
\mathrm{~cm}^{2} / \mathrm{s}\end{array}$ & $\begin{array}{l}R_{h 1}, \\
\mathrm{~nm}\end{array}$ & $\begin{array}{l}R_{h 1}, \\
\mathrm{~nm}\end{array}$ & $\begin{array}{l}\zeta, \\
\mathrm{mW}\end{array}$ \\
\hline 1 & PAMPS-1 & PEC-1 & 2.00 & 0.16 & 12 & 152 & -14.1 \\
2 & PAMPS-1 & IPEC-1 & & 0.14 & & 173 & -20.3 \\
3 & PAMPS-2 & PEC-1 & 0.94 & 0.23 & 26 & 105 & -16.9 \\
4 & PAMPS-2 & IPEC-1 & & 0.21 & & 116 & -33.4 \\
5 & PAMPS-3 & PEC-1 & 0.88 & 0.24 & 28 & 101 & -18.2 \\
6 & PAMPS-3 & IPEC-1 & & 0.25 & & 97 & -50.6 \\
\hline
\end{tabular}


Table 3

Molecular characteristics of PEC-2 and IPEC-2 in $0.05 \mathrm{M} \mathrm{NaCl}$.

\begin{tabular}{llllllr}
\hline Sample & $\begin{array}{l}\mathrm{c}, \\
\mathrm{mM}\end{array}$ & PEC/IPEC & $\begin{array}{l}D_{1} \times 10^{7}, \\
\mathrm{~cm}^{2} / \mathrm{s}\end{array}$ & $\begin{array}{l}D_{2} \times 10^{7}, \\
\mathrm{~cm}^{2} / \mathrm{s}\end{array}$ & $\begin{array}{l}R_{h 1}, \\
\mathrm{~nm}\end{array}$ & $\begin{array}{l}R_{h 1}, \\
\mathrm{~nm}\end{array}$ \\
\hline 1 & 0.77 & PEC-2 & 2.10 & 0.47 & 12 & 52 \\
2 & 0.77 & IPEC-2 & 2.24 & 0.39 & 11 & 63 \\
3 & 1.5 & PEC-2 & 1.71 & 0.52 & 14 & 47 \\
4 & 1.5 & IPEC-2 & & 0.18 & & 135 \\
5 & 3.4 & PEC-2 & 2.38 & 0.72 & 10 & 34 \\
6 & 3.4 & IPEC-2 & Precipitate & & & \\
\hline
\end{tabular}

friction within the framework of the wormlike necklace chain model.

$\frac{\eta_{0} D M}{k T}=P_{\infty}^{-1}[(1-\epsilon)(1-\epsilon / 3)]^{-1} \times M^{\frac{(1-\epsilon)}{2}} A^{\frac{(\epsilon-1)}{2}} M_{L}^{\frac{(\epsilon+1)}{2}}+\frac{M_{L}}{3 \pi}\left[\ln \frac{A}{d}+1+\Psi(\epsilon)\right]$

The value of the $\epsilon=2 v-1$ parameter was calculated from the values of $v(v=0.62)$ obtained experimentally. The $\Psi(\epsilon)$ function is tabulated in [33].

The dependences obtained on the basis of Eqs. (7) and (8) are given in Fig. 4. These dependences were approximated by straight lines. From the value of the intercept (Fig. 4a), the Kuhn segment length was calculated $(A=7 \mathrm{~nm})$. When this value was obtained by approximation of experimental data in accordance with Eq. (8) and from the intercept of the line plotted in Fig. 4b, the $A$ value was $6 \mathrm{~nm}$. Besides, the intercept value (Fig. 4b) was used to determine the effective hydrodynamic diameter of PAMPS-Na macromolecule $(d=0.9 \mathrm{~nm})$. It corresponds to the diameter $d=\zeta / 3 \pi \eta_{0}$ of an element in the wormlike coil model with element friction coefficient $\zeta$ [33].

The authors of [34] analyzed the dependence between the radii of gyration of PAMPS-Na macromolecules and their molecular masses and, taking into account the long-range intrachain interactions, determined the values of persistent length $a_{p}=3 \mathrm{~nm}$ in $0.05 \mathrm{M} \mathrm{NaCl}$ solutions. Using the simple relationship $a_{p}=A / 2$, we can calculate the Kuhn segment length $(A=6 \mathrm{~nm})$. Thus, experimental data obtained by various methods compare very well.

\subsection{Determination of critical aggregation concentration and selection of optimal concentrations for preparing complexes}

The critical aggregation concentration (CAC) was determined for the PEC-1 complex (based on PAMPS-Na macromolecule and surface-active monomer AUTAB) by ionometric analysis using the surfactant-selective electrode according to the technique described in [30]. The dependence of electromotive force (EMF) in electrochemical cell on AUTAB concentration demonstrates two kinks (Fig. 5, points A and B). The A point corresponds to the beginning of cooperative binding between surfactant and polyelectrolyte $(\mathrm{CAC}=0.18 \mathrm{mM}$ ). The $\mathrm{B}$ point corresponds to the saturation concentration $c_{S-A U T A B}$ (the AUTAB concentration where the polymer is saturated with surfactant) and is equal to $0.5 \mathrm{mM}$. Further increase in AUTAB surfactant concentration up to $1 \mathrm{mM}$ leads to an increase in the EMF value and, therefore, to an increase in the amount of free charge carriers. In other words, in the AUTAB concentration range of 0.5 to $1 \mathrm{mM}$, the portion of bound surfactant molecules does not change at a PAMPS-Na concentration of $0.77 \mathrm{mM}$.

Thus, after the addition of a stoichiometric amount of the surfactant ( $C=0.77 \mathrm{mM}$ ), stoichiometric complex is not formed. Polyelectrolyte contains free ionic groups, and the portion of these groups can be estimated from the following relationship: $\left(c_{\text {PAMAPS }}-c_{S-A U T A B}\right) / c_{\text {PAMAPS.; }}$; it is $35 \%$. These conclusions about the structure of the complex are supported by the fact that the values of $\zeta$-potential found for PEC- 1 dispersions are negative (Table 2).

After an increase in polyelectrolyte concentration up to $3.4 \mathrm{mM}$, the addition of a stoichiometric amount of the surfactant results in phase separation in the system and the formation of a precipitate. This behavior is characteristic of stoichiometric complexes [7]. Concentrations of components of the PEC- 2 and IPEC- 2 complexes based on the PAUTAB polyelectrolyte were selected from the same concentration range (Table 3).

In our work, methods of molecular hydrodynamics and atomic force microscopy were used in the studies of complexes obtained at concentrations exceeding CAC and concentrations lower than that for phase separation in the system (Table 3). The studied PECs and IPECs were stable for at least a month. Small-angle X-ray scattering was used in the studies of IPEC films; these IPECs were obtained in the process of PEC polymerization at the concentration exceeding the value which corresponds to phase separation.

\subsection{Polymerization of AUTAB in the presence of PAMPS-Na}

The hydrodynamic radius distributions based on light scattering data for PAMPS-Na samples of various molecular masses include two main peaks (Fig. 6). Diffusion coefficients and the values of hydrodynamic radii calculated from these coefficients are given in Table 2.

Smaller values of hydrodynamic radii (Fig. 6, Table 2) are comparable with the radii of individual PAMPS-Na macromolecules (Table 1). They are also close to the hydrodynamic radii of PEC-1 (PAMPSAUTAB) containing one PAMPS-Na macromolecule. The values of higher hydrodynamic radii (Fig. 6, Table 2) apparently correspond to PEC-1 aggregates. The core of this aggregate may include hydrophobic sequences of the PAMPS-AUTAB complex stabilized with free fragments of PAMPS-Na.

In the case of PEC-1 polymerization at a concentration of $0.77 \mathrm{mM}$, stable dispersion of IPEC- 1 is formed. The spectra of the hydrodynamic radii of stable IPEC-1 included one peak (Fig. 6). The hydrodynamic radii of the particles observed in the solution turned out to be close to these for the PAMPS-AUTAB complex aggregates (which are present in PEC-1 solutions).

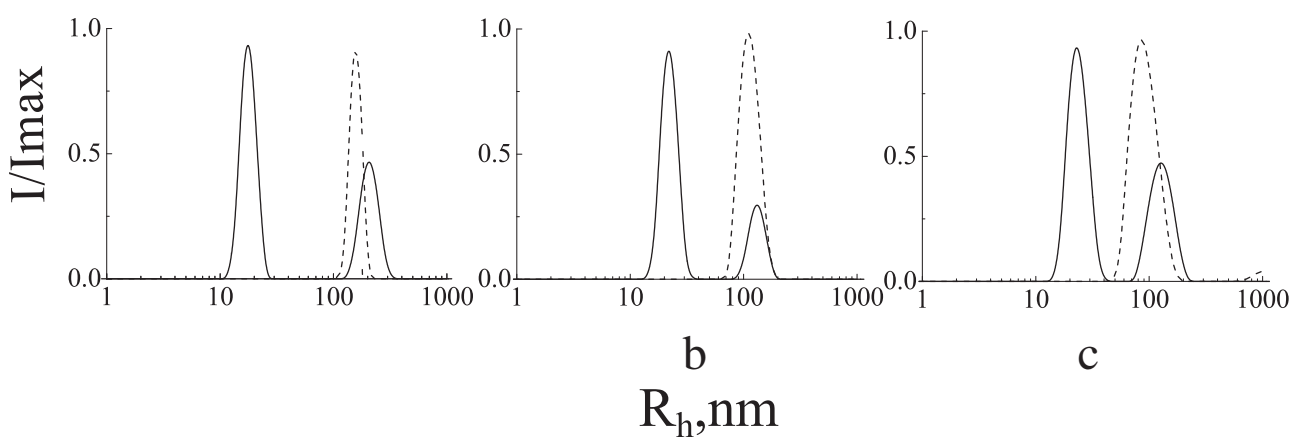

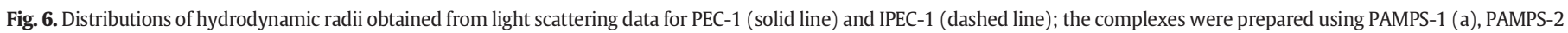
(b) and PAMPS-3 (c) matrices in $0.05 \mathrm{M} \mathrm{NaCl}$ solution. 
$2.8 \mathrm{~nm}$

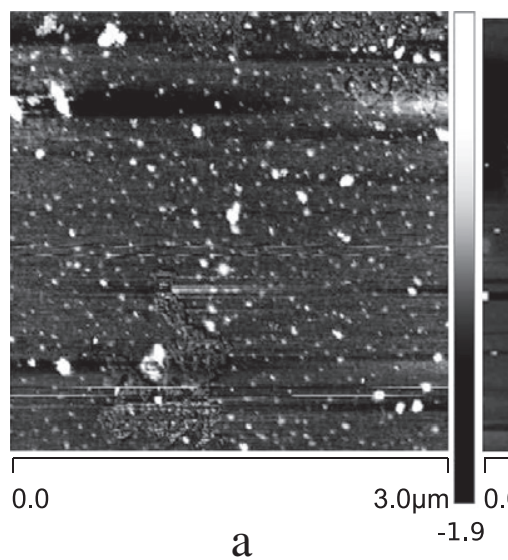

$11.4 \mathrm{~nm}$

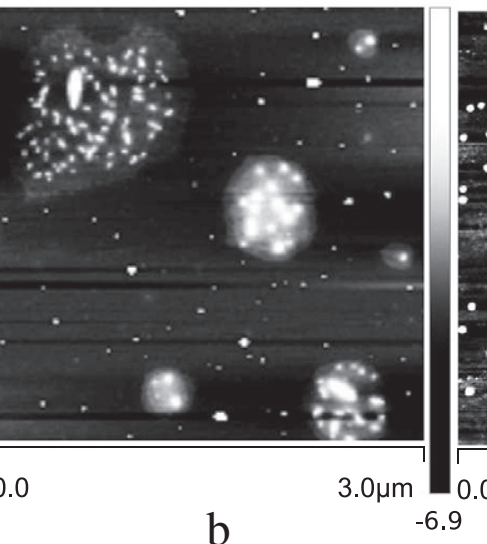

$4.2 \mathrm{~nm}$

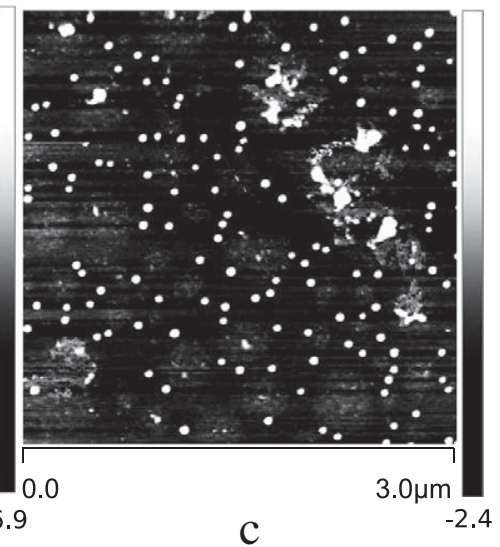

Fig. 7. AFM images of aqueous dispersions of IPEC-1 prepared on the basis of the following matrices: PAMPS-Na-1 (a), PAMPS-Na-2 (b), and PAMPS-Na-3 (c).

Apparently, in the solutions of PEC-1, dynamic equilibrium establishes between the PAMPS-AUTAB complex and its large aggregates. Polymerization of AUTAB included in PEC-1 leads to a shift in this dynamic equilibrium towards the formation of PAMPS-PAUTAB aggregates.

That is, after incorporation into an aggregate, PEC-1 particles are covalently bound to the similar molecules of polymerized surfactant included in other complexes and thus fixate the structure of the formed IPEC-1 particle.

In the hydrodynamic radius distributions obtained for IPEC-1, no particles with the sizes close to those of individual PAMPS-Na molecules were observed, as opposed to the case of PEC-1 solutions (Fig. 6). This fact may indicate that no free PAMPS-Na polyelectrolyte was present in PEC-1 solution.

That is, the peak in the region of smaller hydrodynamic radii is attributed to the PEC-1 complex including one PAMPS-Na macromolecule; the particles characterized by these radii were subsequently included into the IPEC-1 aggregate.

It should be noted that at the AUTAB concentrations selected for IPEC-1 preparation, and in the absence of PAMPS-Na, the attempt to conduct AUTAB polymerization was not completely successful; apparently, this failure was caused by insufficiently high concentration of monomer in the solution. The presence of a polymer matrix leads to a local increase in concentration of surface-active monomer (AUTAB), which facilitates their subsequent covalent binding.

The obtained dispersions of IPEC-1 were visualized using AFM (Fig. 7). The images demonstrate that the particles present on the substrate are spherically symmetric. According to AFM data it is impossible to quantitatively estimate a hydrodynamic radius of polymer particles in solution due to solvent evaporation and sorption of particles. Only qualitative estimation can be made by measuring the particle diameter on a substrate surface. According to AFM data the mean diameters of IPEC-1 particles are 60, 39, and $42 \mathrm{~nm}$, corresponding to Fig. 7a,b,c but their sizes don't agree with the hydrodynamic radii of IPEC-1 estimated by dynamic light scattering (Table 2 ).

Composition of the formed aggregates may be estimated by comparing hydrodynamic radii of various IPECs. The ratio between volumes of complexes including certain amounts of macromolecules $\left(n_{1}\right.$ and $\left.n_{2}\right)$ with the respective degrees of polymerization $Z_{1}$ and $Z_{2}$ may be represented as $V_{2} / V_{1}=\left(n_{2} / n_{1}\right)\left(Z_{2} / Z_{1}\right)^{3 v}$. Water-salt solution is a thermodynamically bad solvent for IPEC-1; therefore, the $v$ parameter is equal to $1 / 3$ [35]. The relationship between amounts of polyelectrolyte matrices included in IPEC-1 may be estimated using the expression $n_{2} / n_{1}=$ $V_{2} Z_{1} / Z_{2} V_{1}$, the values of the IPEC- 1 hydrodynamic radii (Table 2 ) and the known degrees of polymerization of PAMPS-Na (Z), see in Table 1. According to this estimation, the number of PAMPS-Na macromolecules included in IPEC-1 (based on PAMPS-Na-1) is about 50 times higher than that in IPEC-1 based on PAMPS-Na-3; this value for the complex based on PAMPS-Na-2 is 3 times higher than that in IPEC-1 based on PAMPS-Na-3. Thus, it can be concluded that the hydrodynamic radius of the obtained IPEC particle is not determined by molecular mass of its polymeric matrix. At the same time, the ratio between IPEC aggregation numbers $n_{1} / n_{2}$ is proportional to the ratio between masses of PAMPS-Na macromolecules.

The authors of [36] synthesized polyaniline (PANI) in the presence of PAMPS-Na matrices and polystyrene sulfonic acid (PSSA) with molecular masses of $1 \times 10^{6}$ and $7 \times 10^{4}$, respectively. The reaction yielded water-soluble IPEC particles (PAMPS-PANI and PSSA-PANI). It was also revealed that the hydrodynamic radii of the synthesized IPECs virtually do not depend on the molecular mass of the matrix.

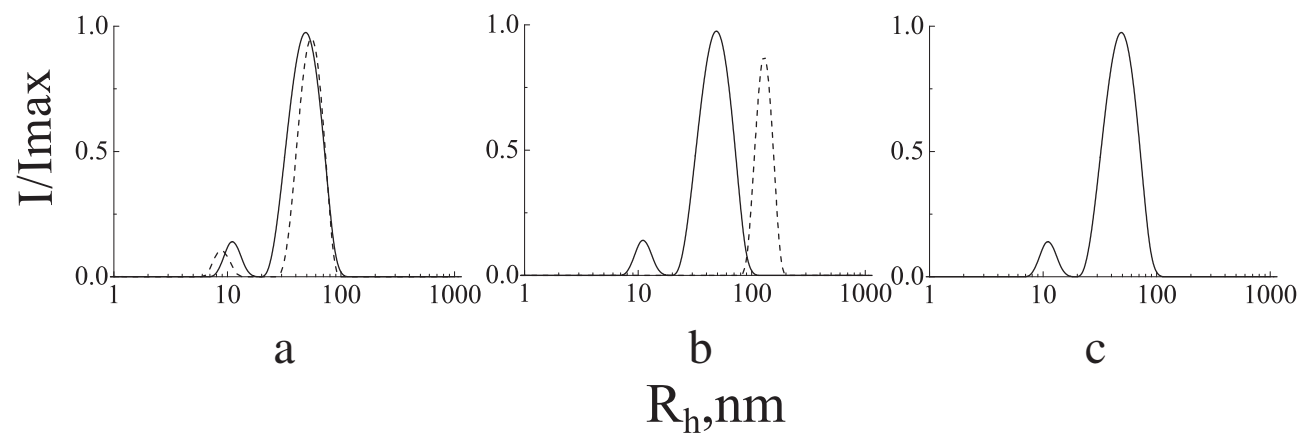

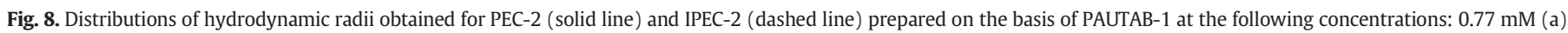
$1.5 \mathrm{mM}$ (b), and $3.4 \mathrm{mM}$ (c) in $0.05 \mathrm{M} \mathrm{NaCl}$ solution. 


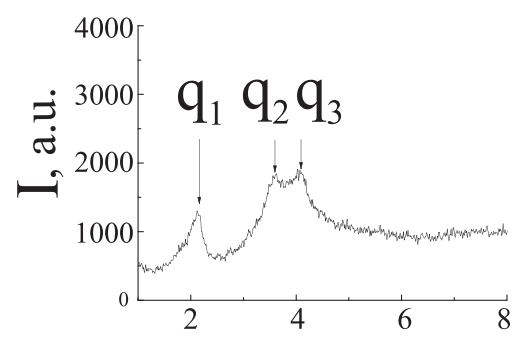

a

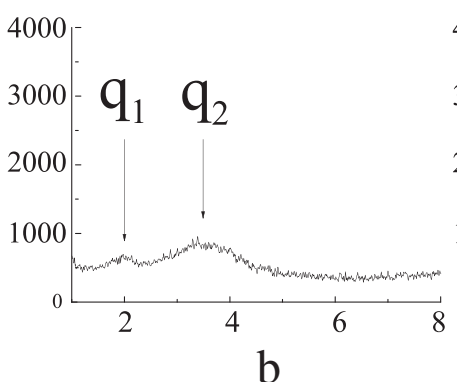

b

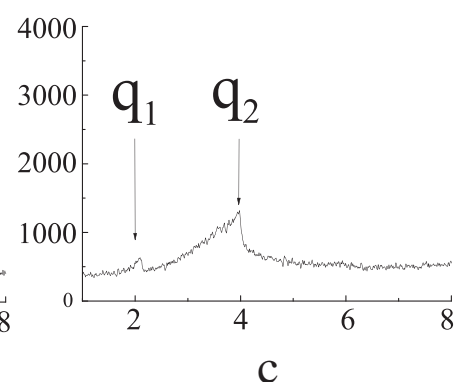

C

\section{$\mathrm{q}, \mathrm{nm}^{-1}$}

Fig. 9. Small-angle X-ray scattering patterns: IPEC-1 (a); IPEC-2 (b); and PAUTAB (c).

\subsection{Polymerization of AMPS in the presence of PAUTAB}

Three solutions of the PEC-2 complex (PAUTAB-AMPS) were prepared at a stoichiometric ratio of components and at various concentrations. The first selected concentration was $0.77 \mathrm{mM}$ (which corresponded to PEC-1 concentration), and the highest studied concentration was $3.4 \mathrm{mM}$.

At a concentration of $0.77 \mathrm{mM}$, in the hydrodynamic radii distributions obtained for PEC-2 solution, two main peaks are present (Fig. 8, Table 3). The position of the first maximum closely corresponds to the value of the hydrodynamic radius of individual PAUTAB macromolecule. The radius corresponding to the second peak was close to the radii of macromolecular aggregates observed in PAUTAB solutions (Table 1, Fig. 2). Bimodal distributions were obtained for PEC-2 dispersions in the whole range of concentrations studied (Fig. 8).

Polymerization at a concentration of $0.77 \mathrm{mM}$ did not cause significant changes in the shape of hydrodynamic radii distributions. This concentration seems to be insufficiently high for AMPS polymerization in the presence of PAUTAB. At a concentration of $1.5 \mathrm{mM}$, after polymerization in the solution of IPEC-2, only one peak in the distribution was observed; the hydrodynamic radius distribution was comparable to that obtained for PEC-1 and IPEC-1 solutions. At a concentration of $3.4 \mathrm{mM}$, polymerization led to phase separation in the system. The study of a supernatant liquid by dynamic light scattering indicated the absence of any particles in this system.

Comparison of the data obtained for IPEC- 1 and IPEC- 2 complexes shows that in the case of IPEC-2, the formation of the interpolyelectrolyte complex with a unimodal hydrodynamic radius distribution requires virtually a two times higher concentration than that in the case of IPEC-1. It is assumed that the important factor in the formation of these complexes is the ability of the AUTAB surfactant included in the complex with PAMPS$\mathrm{Na}$ to form micelle-like aggregates, which facilitates polymerization of this surfactant.

\subsection{The studies of IPEC-1 and IPEC-2 in condensed state}

As stated above, at PEC-1 concentrations exceeding $0.77 \mathrm{mM}$, phase separation occurs in the course of polymerization, and the IPEC-1 precipitate is formed. Polymerization of PEC-2 at concentrations exceeding $1.5 \mathrm{mM}$ also led to phase separation and the formation of the IPEC-2 precipitate. The films of IPEC- 1 and IPEC- 2 were obtained by collecting and drying the precipitates formed after PEC- 1 and $\mathrm{PEC}-2$ polymerization conducted at a concentration of $3.4 \mathrm{mM}$.

According to the small-angle X-ray scattering data (Fig. 9a, b), the IPEC-1 film includes polymerized AUTAB micelles with hexagonal liquid crystal packing (the ratio of scattering vectors $q_{1}: q_{2}: q_{3}=1: 3^{0,5}: 2[2]$ ), and IPEC-2 manifests lamellar packing (the ratio of scattering vectors $q_{1}: q_{2}=1: 2$ [2]). Similar lamellar packing is characteristic of PAUTAB films (Fig. 9c).
Thus, depending on the method used for preparing interpolyelectrolyte complexes, the systems have different morphologies in the condensed state. The use of PAMPS-Na polyelectrolyte as a base allows fixating intramolecular micelles of AUTAB. On the other hand, when PAUTAB macromolecules are used as a base, and AMPS monomer is polymerized, IPECs with a lamellar structure can be obtained (similar to that of PAUTAB films).

\section{Conclusions}

Three samples of anionic polyelectrolyte PAMPS-Na were synthesized by free radical polymerization of AMPS-Na monomer in aqueous solution. Two samples of cationic polyelectrolyte PAUTAB were also prepared. The Mark-Kuhn-Houwink equations for PAMPS-Na polymers dissolved in $0.05 \mathrm{M} \mathrm{NaCl}$ solution were obtained. It was demonstrated that $0.05 \mathrm{M} \mathrm{NaCl}$ solution is a thermodynamically good solvent for PAMPS-Na macromolecules. In PAUTAB solutions (the solvent was also $0.05 \mathrm{M} \mathrm{NaCl}$ solution), polymer aggregation due to hydrophobic interaction of aliphatic tails was observed.

The PAMPS-Na matrices of three different molecular masses and the PAUTAB matrix were used to prepare polyelectrolyte complexes PEC-1 (PAMPS-AUTAB) and PEC-2 (PAUTAB-AMPS) by mixing solutions of corresponding compounds in $0.05 \mathrm{M} \mathrm{NaCl}$ in stoichiometric ratios. Stability of PEC is not only determined by the ratio of solution components, but also it depends on share of surfactant molecules involved in the complex organization. The stability of the PEC- 1 can be adjusted by variation of the total concentration of the components of the system. It is important, that the number of AUTAB surfactant molecules, involved in the complex formation, is independent on molecular weight of PAMPS-Na.

Distributions of hydrodynamic radii of the particles present in the systems were obtained. These distributions were bimodal in the cases of PEC- 1 and PEC-2; it was found that the fast-moving component corresponded to PEC diffusion, and the slow-moving component represented large PEC aggregates. After polymerization of PEC- 1 and PEC-2 at concentrations of $0.77 \mathrm{mM}$ and $1.53 \mathrm{mM}$, respectively, only the particles identified as IPEC aggregates are left in the system. This occurs due to the fact that the polymerization in solutions of PEC- 1 and PEC- 2 shifts the dynamic equilibrium towards the formation of large aggregates of IPEC- 1 and IPEC-2, respectively. For IPEC-1, it was found that the size of the obtained particles is not proportional to the molecular weight of PAMPS-Na macromolecule.

At concentrations of components exceeding $0.77 \mathrm{mM}$ and $1.53 \mathrm{mM}$ for PEC-1 and PEC-2, respectively, polymerization resulted in phase separation. It was revealed that although IPECs have virtually identical chemical structure, film packaging depends on the nature of the polymer base. 


\section{Acknowledgments}

The project was realized with the use of the instruments of resource centers of the Saint-Petersburg State University Research Park (Centre for Diagnostics of Functional Materials for Medicine, Pharmacology and Nanoelectronics, Chemical Analysis and Materials Research Centre, Centre for Optical and Laser Materials Research).

The authors are grateful to the Russian Foundation for Basic Research for the financial support (the projects 14-03-31861 and 1303-00474, Postdoc grant 11.50.1589.2013). This work was also supported by the Saint Petersburg State University research grant (11.38.267.2014).

\section{References}

[1] J. Koetz, S. Kosmella, Polyelectrolytes and Nanoparticles, Springer, 2007.

[2] K. Kogej, Association and structure formation in oppositely charged polyelectrolytesurfactant mixtures, Adv. Colloid Interf. Sci. 158 (2010) 68-83.

[3] L. Piculell, J. Norrman, A. Svensson, I. Lynch, J. Bernardes, W. Loh, Adv. Colloid Interf. Sci. 147 (2009) 228-236.

[4] J. Bernardes, W. Loh, Structure and phase equilibria of mixtures of the complex salt hexadecyltrimethylammonium polymethacrylate, water and different oils, J. Colloid Interface Sci. 318 (2008) 411-420.

[5] B. Ray, S. El Hasri, J.-M. Guenet, Effect of polymer tacticity on the molecular structure of polyelectrolyte/surfactant stoichiometric complexes in solutions and gels, Eur. Phys. J. E 11 (2003) 315-323, http://dx.doi.org/10.1140/epje/i2003-10020-0.

[6] E.N. Govorun, A.S. Ushakova, A.R. Khokhlov, Microphase separation in polymer solutions containing surfactants, Eur. Phys. J. E 32 (2010) 229-242, http://dx.doi.org/10. 1140/epje/i2010-10639-6.

[7] N. Jain, S. Trabelsi, S. Guillot, D. McLoughlin, D. Langevin, P. Letellier, Critical aggregation concentration in mixed solutions of anionic polyelectrolytes and cationic surfactants, Langmuir 20 (2004) 8496-8503.

[8] J. Zakharova, M. Otdelnova, E. Ivleva, et al., Poly( $N$-ethyl-4-vinylpyridinium bromide)-sodium dodecyl sulfate complexes. Formation and supramolecular organization in salt containing aqueous solutions, Polymer 48 (2007) 220-228.

[9] G. Nizri, S. Lagerge, A. Kamyshny, D. Major, S. Magdassi, Polymer-surfactant interactions: binding mechanism of sodium dodecyl sulfate to poly(diallyldimethylammonium chloride), J. Colloid Interface Sci. 320 (2008) 74-81.

[10] D. Langevin, Complexation of oppositely charged polyelectrolytes and surfactants in aqueous solutions. A review, Adv. Colloid Interf. Sci. 147-148 (2009) 170-177.

[11] D. Ganeva, C. Faul, R. Sanderson, Directed reactions within confined reaction environments: polyadditions in polyelectrolyte-surfactant complexes, Macromolecules 36 (2003) 2862-2866.

[12] D. Ganeva, M. Antonietti, C. Faul, R. Sanderson, Polymerization of the organized phases of polyelectrolyte-surfactant complexes, Langmuir 19 (2003) 6561-6565.

[13] K. Chatjaroenporn, R.W. Baker, P.A. FitzGerald, G.G. Warr, Structure changes in micelles and adsorbed layers during surfactant polymerization, J. Colloid Interface Sci. 336 (2009) 449-454.

[14] Soft Matter Characterization, in: R. Borsali, R. Pecora (Eds.), Springer Science + Business Media, LLC, New York, 2008.

[15] Y.V. Shulevich, G. Petzoldb, A.V. Navrotskiia, I.A. Novakova, Properties of polyelectrolyte-surfactant complexes obtained by polymerization of an ionic monomer in a solution of an oppositely charged surfactant, Colloids Surf. A Physicochem. Eng. Asp. 415 (2012) 148-152, http://dx.doi.org/10.1016/j.colsurfa. 2012.10.013.
[16] N. Kasyanenko, L. Lysyakova, R. Ramazanov, A. Nesterenko, I. Yaroshevich, E. Titov G. Alexeev, A. Lezov, I. Unksov, Conformational and phase transitions in DNA-photosensitive surfactant solutions: experiment and modeling, Biopolymers 103 (2) (2015) 109-122, http://dx.doi.org/10.1002/bip.22575.

[17] M. Štěpánek, P. Matějícek, K. Procházka, S.K. Filippov, B. Angelov, M. Šlouf, G. Mountrichas, S. Pispas, Polyelectrolyte-surfactant complexes formed by poly[3,5bis(trimethylammoniummethyl)4-hydroxystyrene iodide]-block-poly(ethylene oxide) and sodium dodecyl sulfate in aqueous solutions, Langmuir 27 (9) (2011) 5275-5281, http://dx.doi.org/10.1021/la200442s.

[18] N.V. Pogodina, N.V. Tsvetkov, Structure and dynamics of the polyelectrolyte complex formation, Macromolecules 30 (17) (1997) 4897-4904.

[19] N.V. Tsvetkov, E.V. Lebedeva, A.A. Lezov, A.N. Podseval'nikova, L.I. Akhmadeeva, M.E Mikhailova, I.M. Zorin, I.A. Makarov, A.Y. Bilibin, Conformational, optical, electrooptical, and dynamic characteristics of cross-linked poly(N-acryloyl-11aminoundecanoic acid), Colloid Polym. Sci. 292 (10) (2014) 2727-2733, http://dx. doi.org/10.1007/s00396-014-3346-1.

[20] N.V. Tsvetkov, E.V. Lebedeva, A.A. Lezov, A.N. Podseval'nikova, L.I. Akhmadeeva, I.M. Zorin, A.Yu. Bilibin, Macromolecules of poly-(12-acryloylaminododecanoic acid) in organic solvent: synthesis and molecular characteristics, Polymer (United Kingdom) 55 (7) (2014) 1716-1723, http://dx.doi.org/10.1016/j.polymer.2014.02.019.

[21] H.Z. Cummins, E.R. Pike (Eds.), Photon-correlation and Light-beating Spectroscopy, Plenum Press, New York and London, 1974.

[22] V.N. Tsvetkov, Rigid-chain Polymers, Consult Bur Plenum NY, London, 1989.

[23] M.L. Huggins, The viscosity of dilute solutions of long-chain molecules. IV. Dependence on concentration, J. Am. Chem. Soc. 64 (11) (1942) 2716-2718, http://dx. doi.org/10.1021/ja01263a056.

[24] V.N. Tsvetkov, E. Eskin, S. Ya Frenkel, Structure of Macromolecules in Solution, National Lending Library for Science and Technology, 1971.

[25] P. Schuck, Size-distribution analysis of macromolecules by sedimentation velocity ultracentrifugation and Lamm equation modeling, Biophys. J. 78 (3) (2000) 1606-1619.

[26] Sedfithomepage http://www.analyticalultracentrifugation.com.

[27] S.W. Provencher, Inverse problems in polymer characterization: direct analysis of polydispersity with photon correlation spectroscopy, Makromol. Chem. 180 (1) (1979) 201-209, http://dx.doi.org/10.1002/macp.1979.021800119.

[28] S.W. Provencher, A constrained regularization method for inverting data represented by linear algebraic or integral equations, Comput. Phys. Commun. 27 (3) (1982) 213-227, http://dx.doi.org/10.1016/0010-4655(82)90173-4.

[29] R. Pamies, J.G. Hernández Cifre, M. del Carmen López Martínez, J. García de la Torre, Determination of intrinsic viscosities of macromolecules and nanoparticles. Comparison of single-point and dilution procedures, Colloid Polym. Sci. 286 (11) (2008) 1223-1231, http://dx.doi.org/10.1007/s00396-008-1902-2.

[30] I. Zorin, T. Scherbinina, P. Fetin, I. Makarov, A. Bilibin, Novel surfactant-selective membrane electrode based on polyelectrolyte-surfactant complex, Talanta 130 (2014) 177-181.

[31] N.V. Tsvetkov, L.N. Andreeva, I.M. Zorin, S.V. Bushin, E.V. Lebedeva, I.A. Strelina, M.A Bezrukova, A.A. Lezov, I.A. Makarov, A.Yu. Bilibin, Synthesis, hydrodynamic, and conformational properties of poly( $\mathrm{N}$-acryloyl-11-aminoundecanoic acid) in solutions, Polym. Sci. Ser. A 53 (5) (2011) 355-363.

[32] J.M.G. Cowie, S. Bywater, The use of frictional coefficients to evaluate unperturbed dimensions in dilute polymer solutions, Polymer 6 (4) (April 1965) 197-204, http://dx.doi.org/10.1016/0032-3861(65)90041-8.

[33] H.B. Gray, V.A. Bloomfield, J.E. Hearst, J. Chem. Phys. 46 (1967) 1493.

[34] J. Yashiro, R. Hagino, S. Sato, T. Norisuye, Chain stiffness and excluded-volume effects in polyelectrolyte solutions: characterization of sodium poly(2-acrylamido-2methylpropanesulfonate) in aqueous sodium chloride, Polym. J. 38 (1) (2006) 57-63.

[35] A.Yu. Grosberg, A.R. Khokhlov, Statistical Physics of Macromolecules, Springer, 1994

[36] Zh.A. Boeva, O.A. Pyshkina, A.A. Lezov, G.E. Polushina, A.V. Lezov, V.G. Sergeyev, Matrix synthesis of water-soluble polyaniline in the presence of polyelectrolytes, Polym. Sci. Ser. C 52 (1) (2010) 35-43, http://dx.doi.org/10.1134/S1811238210010054. 